\title{
PROGRESS
}

\section{Drosophila and the genetics of the internal milieu}

\author{
Pierre Leopold ${ }^{1} \&$ Norbert Perrimon ${ }^{2}$
}

'Homeostasis', from the Greek words for 'same' and 'steady', refers to ways in which the body acts to maintain a stable internal environment despite perturbations. Recent studies in Drosophila exemplify the conservation of regulatory mechanisms involved in metabolic homeostasis. These new findings underscore the use of Drosophila as a model for the study of various human disorders.

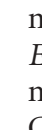
n 1865, Claude Bernard, in his Introduction to the Study of Experimental Medicine, proposed the concept of the 'internal milieu', later referred to as 'homeostasis' in 1932 by Walter Cannon. Homeostasis is one of the most remarkable properties of complex systems that permit organisms to function effectively in a broad range of environmental conditions and allow survival against fluctuations such as temperature, salinity, acidity and nutrients. The kidney, for example, contributes to homeostasis by maintaining salt and ion levels in the blood, regulating the excretion of urea, reabsorbing substances into the blood and regulating blood water levels. Homeostasis depends on the dynamic action and interaction of a number of sensors to adapt to ever-changing environmental conditions, and on hundreds of positive and negative feedback mechanisms.

In recent years, and perhaps surprisingly, we have learned, mostly from genetic studies of physiological responses in Drosophila, that many parallels exist between invertebrate and mammalian homeostasis. As exemplified by the seminal studies of Vincent Wigglesworth ${ }^{1}$, insects have historically been long-standing models in physiology as a result of their technical advantages such as short life cycles, large populations and the possibility of simple surgical procedures. In the past, however, interest in their physiology was driven mostly by intellectual fascination with their diverse physiological adaptations to virtually every habitat on Earth, as well as by potential applications for pest control. Here we review several recent findings that establish Drosophila as an emerging model for mammalian physiology.

\section{Metabolic homeostasis}

Drosophila and all other higher organisms constantly adapt their energy needs to nutritional status through metabolic regulation; that is, sugar and lipid homeostasis. The emerging picture in the fly is that of a simpler and well-balanced integrated system between various organs, each with distinct physiological roles in maintaining energy homeostasis (Fig. 1). Drosophila has a less complex genome than vertebrates and has little gene redundancy. For example, flies have a unique insulin receptor that mediates all functions that have so far been attributed to insulin-like peptides. Sugar levels are maintained by neurosecretory cells located in the brain and ring gland (together forming a bipartite 'Drosophila pancreas') that secrete insulin and adipokinetic hormone ( $\mathrm{AKH}$, the insect glucagon) into the open haemolymph (the 'Drosophila blood') ${ }^{2-5}$ (reviewed in refs 6-8). Excess sugar is stored in the form of glycogen that accumulates both in muscles and in the fat body (the 'Drosophila liver'). On stimulation by $\mathrm{AKH}$, the fat-body glycogen phosphorylase is activated and sugar (trehalose in insects) is released into the haemolymph. The fat body also acts as the storage place for fat and thus is reminiscent of the 'white fat' of vertebrates. A recent series of studies illustrate the convergences in the control of lipid metabolism between flies and mammals. When food is scarce, fat is released from the fat body into the haemolymph and is then captured by the oenocytes (the fly 'hepatocytes') for energy production'. In both systems, information on energy shortage is relayed by lipolytic hormones, which activate a pathway dependent on cyclic-AMP-dependent protein kinase that leads to the activation of specific lipases at the surface of intracellular lipid droplets. However, in flies the specific role of these lipases is still unclear: both adipose triglyceride lipase (ATGL) knockout mice and brummer (the ATGL orthologue in flies) mutant flies are obese as a result of an impairment of lipid mobilization ${ }^{10,11}$.

Genetic ablations have had major roles in deciphering the elaborate crosstalk between the various sensor tissues and their responsive organs. For example, ablation of insulin-producing cells leads to diabetic flies and, conversely, flies in which AKH-producing cells have been ablated have low levels of circulating sugars ${ }^{3,12}$. Further, proper mobilization of fat from the fat body is perturbed in the absence of oenocytes - an interaction reminiscent of the crosstalk between hepatocytes and adipocytes in humans ${ }^{9,13}$. Additional

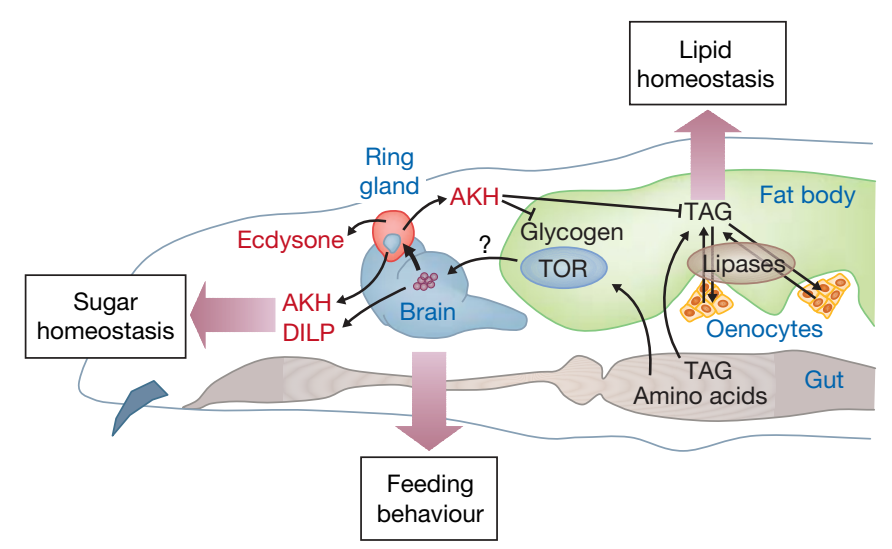

Figure 1 | Interactions between the various organs involved in metabolic homeostasis in a Drosophila larva. Hormone names are shown in red, tissue names in blue. DILP, Drosophila insulin-like peptides; TAG: triacylglycerol. 
insights have been obtained from the use of drugs such as sulphonylurea (glyburide or tolbutamide), currently used in the treatment for type 2 diabetes as activators of insulin secretion by pancreatic beta cells ${ }^{14}$. Drosophila larvae fed with sulphonylurea show increased sugar levels, suggesting that AKH-expressing cells in the fly use a conserved mechanism for coupling circulating sugars to glucagon and $\mathrm{AKH}$ release ${ }^{4}$.

An important notion emerging from studies in the fly is that all tissues in the organism might not be exposed or equally sensitive to variations in nutritional conditions. The fat body, in particular, seems to be a unique metabolic sensor because it has a pivotal function in buffering nutritional information for the entire animal ${ }^{15-17}$. For example, in conditions of amino-acid restriction, the conserved TOR pathway in fat cells functions as an energy sensor that induces a remote and systemic inhibition of insulin/insulin-like growth factor signalling, leading to a general decrease in growth during larval development ${ }^{16}$.

Cross-regulation between tissues for the control of insulin signalling is reminiscent of recent observations in mammals related to insulin resistance, which leads to obesity and type 2 diabetes, two components of a highly prevalent and complex disorder called the metabolic syndrome ${ }^{18,19}$. Whether Drosophila is a good model for dissecting the basic molecular and genetic mechanisms of this disorderis still uncertain; however, the first example of insulinresistant flies has emerged recently. Teleman et al. ${ }^{20}$ showed that flies that do not express the microRNA-encoding gene $m i R-278$ in the fat body are lean and have elevated levels of sugar in the haemolymph, despite high expression levels of insulin. Further, whereas injection of insulin into wild-type larvae provoked an activation of the insulin pathway in fat cells, this response was abrogated in $m i R-278$ mutant animals, indicating that the 'lean fly' phenotype is associated with insulin resistance in the fat body ${ }^{20}$. These findings are reminiscent of the decreased fat-pad mass observed in the fat-specific insulin receptor knockout model in the mouse ${ }^{21}$. Thus, as in mammals, insulin signalling in Drosophila regulates patterns of energy storage in fat and other tissues, and disruption of this process by insulin resistance leads to important lipid disorders.

\section{Interaction between metabolism and other processes}

In addition to progress in our understanding of the logic of metabolic homeostasis in the fly, important advances are being made in our knowledge of the complex interactions between metabolism and growth, ageing or behaviour. For relevant reviews in the Drosophila literature see refs 22-25. How organisms adapt their growth programme to changes in energy needs and status, and how hormonal systems such as insulin and steroid hormones interact with metabolic regulation, are areas of particular interest for future research. In particular, functional interactions recently identified between insulin and the steroid hormone ecdysone $e^{26,27}$ suggest that fly steroids also participate in metabolic control. Further, in adult flies a decrease in metabolic rates resulting from dietary restriction or decreased insulin signalling results in extension of the normal lifespan (reviewed in refs 23,24$)$. The mechanism by which dietary restriction controls lifespan is unclear, but metabolic studies in this short-lived model system will undoubtedly contribute to an explanation of the control of ageing by nutrition.

Finally, several complex behaviours are modulated by metabolic conditions. Feeding behaviour is regulated by two major intermingled controls: the homeostatic and the hedonic systems ${ }^{28}$. Homeostatic regulation of feeding ensures that circulating nutrient levels are sensed, which in turn directly regulates feeding activity. Work on Drosophila and studies on the mouse recently showed that a TOR-dependent molecular sensor for amino acids exists in specific brain cells (hypothalamic cells in the mouse; neuroendocrine cells in Drosophila). These cells sense variations in circulating amino-acid levels and in turn regulate feeding ${ }^{29,30}$. Understanding the circuits that transmit these neuroendocrine signals is of prime interest for the study of eating disorders and are beginning to be studied in Drosophila ${ }^{25}$.

\section{Concluding remarks}

The goal of this review is to provide a selective overview of recent progress in studies of metabolic homeostasis in Drosophila. Through the examples cited, it is clear that many questions will be answered in the next few years. For example, studies will decipher the specific crosstalk between various larval and adult tissues for the control of energy homeostasis. Characterization of mutants associated with insulin resistance is likely to provide insights into the mechanisms of insulin response and its disruption. In addition, Drosophila has great potential as a model system in which to study the balance between lipid storage and lipolysis, a process whose disruption in humans leads to obesity or type 2 diabetes.

In flies, the characterization of homeostatic regulatory mechanisms is facilitated by the smaller number of homeostatic constants and, of course, the ever-improving arsenal of available genetic tools. Genetic screens have already identified new components of metabolic regulatory networks (such as melted, a new modulator of FOXO and TSC2 activity $^{31}$ ), whose function can now be tested in mammals. Genome-wide RNA-mediated interference (RNAi) screens, both in tissue culture cells ${ }^{32}$ and in vivo ${ }^{33}$, provide unique tools for the study of metabolic regulations. In particular, in vivo RNAi, allowing the activity of a specific pathway to be reduced in a given tissue, should showcase Drosophila as a model for metabolic disorders. These Drosophila models should prove to be very powerful for drug screening, to determine modes of drug action, and to identify drug targets. Finally, a better characterization of Drosophila metabolites will benefit the continuing work to understand experimental perturbations of the 'internal milieu'.

1. Wigglesworth, V. B. Principles of Insect Physiology (Chapman \& Hall, London, 1972).

2. Brogiolo, W. et al. An evolutionarily conserved function of the Drosophila insulin receptor and insulin-like peptides in growth control. Curr. Biol. 11, 213-221 (2001).

3. Rulifson, E. J., Kim, S. K. \& Nusse, R. Ablation of insulin-producing neurons in flies: growth and diabetic phenotypes. Science 296, 1118-1120 (2002).

4. Kim, S. K. \& Rulifson, E. J. Conserved mechanisms of glucose sensing and regulation by Drosophila corpora cardiaca cells. Nature 431, 316-320 (2004).

5. Lee, G. \& Park, J. H. Hemolymph sugar homeostasis and starvation-induced hyperactivity affected by genetic manipulations of the adipokinetic hormoneencoding gene in Drosophila melanogaster. Genetics 167, 311-323 (2004).

6. Gade, G., Hoffmann, K. H. \& Spring, J. H. Hormonal regulation in insects: facts, gaps, and future directions. Physiol. Rev. 77, 963-1032 (1997).

7. Wu, Q. \& Brown, M. R. Signaling and function of insulin-like peptides in insects. Annu. Rev. Entomol. 51, 1-24 (2006).

8. Geminard, C. et al. Control of metabolism and growth through insulin-like peptides in Drosophila. Diabetes 55 (Suppl. 2), S5-S8 (2006).

9. Gutierrez, E., Wiggins, D., Fielding, B. \& Gould, A. P. Specialized hepatocyte-like cells regulate Drosophila lipid metabolism. Nature 445, 275-280 (2007).

10. Gronke, S. et al. Brummer lipase is an evolutionary conserved fat storage regulator in Drosophila. Cell Metab. 1, 323-330 (2005).

11. Haemmerle, G. et al. Defective lipolysis and altered energy metabolism in mice lacking adipose triglyceride lipase. Science 312, 734-737 (2006).

12. Broughton, S. J. et al. Longer lifespan, altered metabolism, and stress resistance in Drosophila from ablation of cells making insulin-like ligands. Proc. Natl Acad. Sci. USA 102, 3105-3110 (2005).

13. Oike, Y., Akao, M., Kubota, Y. \& Suda, T. Angiopoietin-like proteins: potential new targets for metabolic syndrome therapy. Trends Mol. Med. 11, 473-479 (2005)

14. Nagashima, K. et al. Sulfonylurea and non-sulfonylurea hypoglycemic agents: pharmacological properties and tissue selectivity. Diabetes Res. Clin. Pract. 66 (Suppl 1), S75-S78 (2004)

15. Britton, J. S. \& Edgar, B. A. Environmental control of the cell cycle in Drosophila: nutrition activates mitotic and endoreplicative cells by distinct mechanisms. Development 125, 2149-2158 (1998).

16. Colombani, J. et al. A nutrient sensor mechanism controls Drosophila growth. Cell 114, 739-749 (2003)

17. Zinke, I., Kirchner, C., Chao, L. C., Tetzlaff, M. T. \& Pankratz, M. J. Suppression of food intake and growth by amino acids in Drosophila: the role of pumpless, a fat body expressed gene with homology to vertebrate glycine cleavage system. Development 126, 5275-5284 (1999).

18. Petersen, K. F. et al. Inaugural article: The role of skeletal muscle insulin resistance in the pathogenesis of the metabolic syndrome. Proc. Natl Acad. Sci. USA 104 12587-12594 (2007). 
19. Grundy, S. M. Metabolic syndrome: a multiplex cardiovascular risk factor. J. Clin. Endocrinol. Metab. 92, 399-404 (2007).

20. Teleman, A. A., Maitra, S. \& Cohen, S. M. Drosophila lacking microRNA miR-278 are defective in energy homeostasis. Genes Dev. 20, 417-422 (2006).

21. Kitamura, T., Kahn, C. R. \& Accili, D. Insulin receptor knockout mice. Annu. Rev. Physiol. 65, 313-332 (2003)

22. Edgar, B. A. How flies get their size: genetics meets physiology. Nature Rev. Genet 7, 907-916 (2006).

23. Giannakou, M. E. \& Partridge, L. Role of insulin-like signalling in Drosophila lifespan. Trends Biochem. Sci. 32, 180-188 (2007).

24. Tatar, M. Diet restriction in Drosophila melanogaster. Design and analysis. Interdiscipl. Top. Gerontol. 35, 115-136 (2007).

25. Melcher, C., Bader, R. \& Pankratz, M. J. Amino acids, taste circuits, and feeding behavior in Drosophila: towards understanding the psychology of feeding in flies and man. J. Endocrinol. 192, 467-472 (2007)

26. Colombani, J. et al. Antagonistic actions of ecdysone and insulins determine final size in Drosophila. Science 310, 667-670 (2005).

27. Mirth, C. Ecdysteroid control of metamorphosis in the differentiating adult leg structures of Drosophila melanogaster. Dev. Biol. 278, 163-174 (2005).

28. Saper, C. B., Chou, T. C. \& Elmquist, J. K. The need to feed: homeostatic and hedonic control of eating. Neuron 36, 199-211 (2002).
29. Wu, Q., Zhang, Y., Xu, J. \& Shen, P. Regulation of hunger-driven behaviors by neural ribosomal 56 kinase in Drosophila. Proc. Natl Acad. Sci. USA 102 13289-13294 (2005)

30. Cota, D. et al. Hypothalamic mTOR signaling regulates food intake. Science 312 927-930 (2006)

31. Teleman, A. A., Chen, Y. W. \& Cohen, S. M. Drosophila Melted modulates FOXO and TOR activity. Dev. Cell 9, 271-281 (2005).

32. Perrimon, N. \& Mathey-Prevot, B. Applications of high-throughput RNA interference screens to problems in cell and developmental biology. Genetics 175, 7-16 (2007)

33. Dietzl, G. et al. A genome-wide transgenic RNAi library for conditional gene inactivation in Drosophila. Nature 448, 151-156 (2007).

Acknowledgements We thank Liz Perkins for comments on the manuscript. Work in the Perrimon laboratory is supported by the Howard Hughes Medical Institute and the $\mathrm{NIH}$, and in the Leopold laboratory by INSERM, the CNRS, the Agence Nationale de la Recherche and the Fondation pour la Recherche Médicale.

Author Information Reprints and permissions information is available at www.nature.com/reprints. Correspondence should be addressed to P.L. (leopold@unice.fr) or N.P. (perrimon@receptor.med.harvard.edu). 